\title{
Identification of filamentous Fungi Isolated from Clinical Specimens by MALDITOF Mass Spectrometry in A Tertiary Health Care Centre
}

\author{
Hena Butta ${ }^{1 *}$, Raman Sardana ${ }^{1}$, Ashmita Grover ${ }^{2}$, Leena Mendiratta ${ }^{1}$, Sudha Kansal $^{3}$ and Neerav Goyal ${ }^{4}$ \\ ${ }^{1}$ Department of Microbiology, Indraprastha Apollo Hospitals, New Delhi, India \\ Department of Microbiology, Indraprastha Apollo Hospitals, New Delhi, India \\ ${ }^{2}$ Department of Microbiology, Sardar Bhawan Singh Postgraduate Institute of Biomedical Sciences and Research, \\ Balawala, Dehradun, Uttrakhand, India \\ Department of Microbiology, Indraprastha Apollo Hospitals, New Delhi, India \\ ${ }^{3}$ Department of Critical Care, Respiratory and Sleep Medicine Indraprastha Apollo Hospitals, New Delhi, India \\ ${ }^{4}$ Institutes of Transplant, Indraprastha Apollo Hospitals, New Delhi, India
}

\begin{abstract}
Background: Matrix Assisted Laser Desorption Ionisation- Time of Flight Mass Spectrometry (MALDI-TOF MS) has revolutionized the identification of microorganisms particularly bacteria and yeasts in terms of rapidity, cost-effectiveness, automation and enhanced discriminatory power. Its utility for the identification of filamentous fungi lags due to certain challenges like effective protein extraction method and limited databases. The conventional methods for the identification of filamentous fungi are time consuming and may not establish the definitive species identification. Keeping this in mind, we planned this study to evaluate MALDI-TOF Vitek MS (Biomerieux, France) for the identification of filamentous fungi, to standardize the mould identification protocol and to correlate the identification results by MALDI TOF MS with the conventional phenotypic methods.

Methods: The identification of clinically significant filamentous fungi isolated from various clinical specimens was done by both conventional method and MALDITOF Vitek MS using Ethanol-Formic acid -Acetonitrile protocol. The unidentified fungi by both the methods were identified in the reference Mycology laboratory.

Result: Out of 50 clinically significant moulds isolated from various clinical specimens during study period, 35 (70\%) could be identified using MALDI-TOF Vitek MS and all the identification results corroborated with the phenotypic method for identification with no misidentification. In addition to Aspergillus species, the identification of Zygomycetes and Phaeoid fungi could also be possible using MALDI TOF Vitek MS.

Conclusion: MALDITOF MS was found to be a rapid and reliable method for the species identification of filamentous fungi. We could standardize two extraction protocols using Ethanol-Formic acid -Acetonitrile at our institute for the identification of filamentous fungi by MALDI TOF Vitek MS. We also emphasize on the creation of own in-house library for the optimum utilization of MALDI TOF Vitek MS for the identification of filamentous fungi.
\end{abstract}

Keywords: MALDI-TOF Vitek MS, Filamentous Fungi, Ethanol-Formic Acid-Acetonitrile Extraction, Macromorphological, Identification

\section{Introduction}

Filamentous fungi (Moulds) are mainly opportunistic pathogens and are a great threat for immunocompromised patients with impaired host defense mechanism. ${ }^{1}$ They are also the important cause of mortality and morbidity among hospitalised patients. ${ }^{2,3}$ The identification of these filamentous fungi is important to manage the growing number of invasive mould infections.,5 Importantly accurate species discrimination of filamentous fungi is essential because some species have specific antifungal susceptibility patterns and misidentifications may lead to inappropriate therapy.

The macromorphological methods for the identification are time consuming and many-a-times do not lead to definitive species identification and prone to error. ${ }^{6}$ Moreover, in the last decade, numerous new species and species complexes of fungi have emerged which have been found to be resistant to antifungal drugs or display a typical susceptibility profile or are phenotypically similar but genetically and possibly pathogenically different from their counterparts. ${ }^{7}$ Indeed the rates of certain mould infections have increased, such as Aspergillosis, Fusariosis and Mucormycosis. ${ }^{4}$ Such factors have complicated the accuracy of traditional phenotypic mould identification for which sufficient growth and sporulation are required to assess macromorphological criteria; including growth on different media, at different temperatures as well as shape of conidia, spores and mycelial structures. The limitations of the conventional methods include long time to result, 
various morphological variations of moulds requiring extensive individual expertise of the laboratory personnel and in some cases moulds do not develop their distinctive structural features under laboratory conditions which may lead to misidentification or un-identification. Such factors have complicated the accuracy of traditional mould identification compelling laboratories to adopt a fast and reliable identification methods for moulds to curtail the morbidity and lethality associated with the filamentous fungi particularly in immunocompromised persons. ${ }^{8,9}$

Matrix assisted laser desorption/ time of flight mass spectrometry (MALDI- TOF MS) has revolutionised the identification of bacteria and yeasts in terms of turnaround time, reliability and economy. MALDI-TOF MS has been found to be a powerful, reproducible, accurate diagnostic tool with enhanced discriminatory power to identify microbial agents. ${ }^{7}$ It analyses the protein content of an unknown isolate under the form of a species specific spectrum typically between 2 and $20 \mathrm{kDa}$, which is then identified with the reference specific spectra of a database. ${ }^{2}$ This technology uses a simple, fast, and a cost effective protocol allowing the identification of any species, provided that it is present in the library. ${ }^{10}$ The identification of moulds by MALDI TOF MS has lagged due to challenges in developing an efficient protein extraction method to achieve good quality mass spectra as compared to that of bacteria because the cell wall of moulds is more robust and rigid making sample preparation more time and labour intensive. ${ }^{7,11}$ But, this technique has now been standardized to a great extent for the identification of filamentous fungi and has been found comparable to molecular techniques like DNA sequencing.

Various studies already conducted shows that MALDI TOF MS holds promise as a fast and accurate identification tool, particularly with common species or typical strains of filamentous fungi. In literature, Bruker MALDI-TOF MS (Bruker Daltonics) has been found to be superior for the identification of filamentous fungi by MALDITOF MS technique and the studies on the identification of filamentous fungi by MALDI-TOF MS have been conducted mainly using Bruker MALDI-TOF MS., 2,711,12,13 So, we have planned this study to evaluate Vitek MS (Biomerieux, France), which is considered superior for the identification of bacteria including Mycobacteria and yeasts and also being used in our laboratory for the routine identification of bacteria and yeasts, for the identification of filamentous fungi. ${ }^{12}$ The primary aims and objectives of the study were to identify the mycelial fungal isolates from various clinical specimens using MALDI TOF Vitek MS, to standardize the mould identification protocol using MALDI TOF Vitek MS at our laboratory and to correlate the identification results by MALDI TOF Vitek MS with the conventional phenotypic method for identification of moulds. The secondary objectives of the study were to initiate antifungal therapy optimally and timely after accurate species identification in shorter time.

\section{Materials And Methods}

The study was conducted in the Department of Microbiology at a tertiary health care centre in New Delhi from November 2016 to April 2016. The clinical specimens received for fungal culture were processed as per the standard laboratory protocol i.e. these were inoculated on two Sabouraud's dextrose agar (SDA) slants and were kept at $25^{\circ}$ and $37^{\circ} \mathrm{C}$ incubators and were observed daily for the growth of any filamentous fungi for a maximum period of three weeks. The correlation of the growth was done with fungal smear examination, histopathological examination and clinical details. The identification of the growth was done by LPCB (Lacto phenol cotton blue staining) mount examination. Slide cultures on SDA/cornmeal agar (CMA)/Potato dextrose agar (PDA) blocks were also put for definitive species identification.

The identification of 50 clinically significant filamentous fungi isolated during study period was done by both conventional methods and MALDI TOF Vitek MS using Ethanol-Formic acid -Acetonitrile extraction method. The spots on Vitek MS target slide were made in duplicate after extraction and allowed to dry. Then, $1 \mu 1$ of CHCA $(\alpha-$-cyano4-hydroxycinnamic acid) matrix was added on the spots and allowed to dry. Escherichia coli (ATCC 8739) reference strain was spotted as a calibrator in each acquisition area. Then, the target slide was processed in MALDI-TOF Vitek MS and analysis of the spectra was performed by Vitek MS MS-ID database for In-vitro Diagnostics. For those isolates, whose results were not obtained with Vitek MS MS-ID database for In-vitro Diagnostics, the identification was done by RUO spectra created for analysis by the SARAMIS database for Research use only.

\section{Sample preparation for MALDI-TOF Vitek MS:}

The extraction of protein of filamentous fungi was done initially by extraction protocol- $1 .{ }^{14}$ For few of the isolates which could not be identified by protocol-1, the other protocol (extraction protocol-2) which is the modified version of extraction protocol by Singh et alwas used. ${ }^{15}$ These protocols are as described below:

Extraction protocol-1: Fungal colonies grown on SDA were taken and mixed in $900 \mu \mathrm{l}$ ethanol in an eppendorf tube. It was then vortexed and centrifuged at 10,000 revolution per minute (rpm)for 2 minutes. The supernatant was discarded and $40 \mu \mathrm{l}$ of freshly prepared $70 \%$ formic acid was added 
and after vortexing again $40 \mu \mathrm{l}$ of acetonitrile was added and centrifuged again at 10,000 rpm for 2 minutes. 1 microlitre of the supernatant from the centrifuged mixture was put onto the target slide and left for drying. 1 microlitre of CHCA matrix was put onto it and the slide was loaded into VITEK MS for further identification.

Extraction protocol-2: Fungal isolates were grown in 9 ml Sabouraud's Dextrose (SD) broth prepared in-house for $24-36$ hours at $25^{\circ} \mathrm{C}$. The SD broth tubes with growth were kept consecutively on rotatory shaker for 10 minutes and then centrifuged at 3,000 rpm for 10 minutes. This procedure was repeated intermittently every two hours for a period of 36 hours. After 36 hours, $1 \mathrm{ml}$ of the culture growth from SD broth tube was taken in eppendorf tube and centrifuged at 14,000rpm for $2 \mathrm{~min}$. The supernatant was discarded and the pellet was washed twice with sterile water. The pellet was then dissolved in 300 microlitre $(\mu 1)$ of sterile water and $900 \mu \mathrm{l}$ of absolute alcohol and vortexed. It was then again centrifuged at 14,000 rpm for 2 minutes. Supernatant was then discarded and $50 \mu \mathrm{l}$ of $70 \%$ formic acid was added and left upon for incubation at room temperature for 2 minutes. $50 \mu \mathrm{l}$ of acetonitrile was then added and left for incubation at room temperature for 5 minutes. This mixture was then centrifuged at $10000 \mathrm{rpm}$ for 2 minutes and $1 \mu 1$ of supernatant was applied on target plate and air dried. $1 \mu \mathrm{l} \mathrm{CHCA} \mathrm{matrix} \mathrm{was} \mathrm{then} \mathrm{applied}$ onto the target spots and analysed by VITEK MS.

The identification results by MALDI TOF Vitek MS and macromorphological methods were compared and analysed.

\section{Result}

The identification of a total of 50 clinical isolates of filamentous fungi was done using MALDI TOF Vitek MS during study period. The maximum number of filamentous fungi were grown from respiratory specimens (26), followed by paranasal sinus (10), subcutaneous pus/ tissue (09), ear pus (03) and cutaneous specimens (02). The maximum number of filamentous fungi isolated from various clinical specimens were Aspergillus species (36), followed by Zygomycetes (08), Phaeohyphomycetes (05) and Dermatophytes (01). Amongst all Aspergillus species (36), A.flavus was isolated maximally (20), followed by A. fumigatus (12). The frequency of occurrence of various moulds from different clinical specimens is described in table-1. The results of MALDI TOF MS and morphological identifications are presented in table-2. Few isolates which were not identified by extraction protocol-1 i.e. after growing the fungi on SDA were grown on SD broth and extraction was done as per protocol-2. The identification could be done in three out of four such isolates.

Table 1: Frequency of occurrence of filamentous fungi from various clinical specimens.

\begin{tabular}{|c|c|c|}
\hline Specimen & Type of mould & Number (\%) \\
\hline \multirow{11}{*}{ Respiratory specimen } & Aspergillus species & \multirow{6}{*}{21} \\
\hline & A. fumigatus (8) & \\
\hline & A.flavus (10) & \\
\hline & A. niger (1) & \\
\hline & A. terreus (1) & \\
\hline & A. nidulans (1) & \\
\hline & Zygomycetes & \multirow{5}{*}{04} \\
\hline & Rhizopus arrhizus complex(1) & \\
\hline & Rhizopus microsporus complex(1) & \\
\hline & Rhizomucor sp.(1) & \\
\hline & Unidentified-1 & \\
\hline \multirow{5}{*}{ Subcutaneous pus /tissue } & Aspergillus species & \multirow{2}{*}{03} \\
\hline & A. fumigatus (03) & \\
\hline & Zygomycetes & \multirow{3}{*}{03} \\
\hline & Mucor sp. -01 & \\
\hline & Unidentified-02 & \\
\hline
\end{tabular}




\begin{tabular}{|c|c|c|}
\hline Specimen & Type of mould & Number (\%) \\
\hline \multirow{4}{*}{ Subcutaneous pus /tissue } & Phaeohyphomycetes & \multirow{4}{*}{03} \\
\hline & Cladophialophora bantiana-01 & \\
\hline & Alternaria alternata-01 & \\
\hline & Unidentified -01 & \\
\hline \multirow{4}{*}{ Cutaneous specimen } & Aspergillus species & \multirow[t]{2}{*}{01} \\
\hline & Aspergillus flavus-01 & \\
\hline & Dermatophytes & \multirow{2}{*}{01} \\
\hline & Microsporum gypseum /cookie-01 & \\
\hline \multirow{8}{*}{ Paranasal sinus pus /tissue } & Aspergillus species & \multirow{3}{*}{08} \\
\hline & Aspergillus fumigatus -01 & \\
\hline & Aspergillus flavus-07 & \\
\hline & Zygomycetes & \multirow{3}{*}{02} \\
\hline & Syncephalastrum racemosum-01 & \\
\hline & Rhizopus arrhizus-01 & \\
\hline & Phaeohyphomycetes & \multirow{2}{*}{01} \\
\hline & Scedosporium apiospermum (01) & \\
\hline \multirow{3}{*}{ Ear pus } & Aspergillus species & \multirow{3}{*}{03} \\
\hline & Aspergillus niger-01 & \\
\hline & Aspergillus flavus-02 & \\
\hline
\end{tabular}

Table 2: Comparison between identification results by conventional method and MALDI TOF Vitek MS.

\begin{tabular}{|c|c|c|}
\hline Name of the filamentous fungi & Conventional identification & MALDITOF Vitek MS identification \\
\hline \multirow{5}{*}{ Aspergillus sp.(36) } & Aspergillus flavus (20) & Aspergillus flavus (16) \\
\hline & Aspergillus fumigatus (12) & Aspergillus fumigatus (09) \\
\hline & Aspergillus niger (02) & Aspergillus niger (02) \\
\hline & Aspergillus terreus (01) & Aspergillus terreus (01) \\
\hline & Aspergillus nidulans (01) & Aspergillus nidulans (01) \\
\hline \multirow{5}{*}{ Zygomycetes (08) } & \multirow{2}{*}{ Rhizopus sp. (03) } & Rhizopus arrhizus complex(02) \\
\hline & & Rhizopus microsporus complex(01) \\
\hline & Syncephalastrum racemosum (01) & Unidentified \\
\hline & \multirow{2}{*}{ Unidentified (04) } & Rhizomucor pussilus (01) \\
\hline & & Unidentified (03) \\
\hline \multirow{4}{*}{ Phaeohyphomycetes (05) } & Cladophialophora species (01) & Cladophialophora bantiana (01) \\
\hline & Alternaria species (01) & Alternaria alternata (01) \\
\hline & Exserohilum rostratum (01) & Unidentified (01) \\
\hline & Scedosporium sp. (02) & Unidentified (02) \\
\hline Dermatophytes (01) & Microsporum gypseum/cookie (01) & Unidentified (01) \\
\hline
\end{tabular}




\section{Discussion}

The present study was conducted in the department of Microbiology, Indraprastha Apollo Hospitals, New Delhi from November 2016 to April 2017. A total of 50 moulds isolated from various clinical specimens were subjected to identification by using MALDI TOF Vitek MS. Out of 50 moulds, 35 (70\%) could be identified using MALDI-TOF Vitek MS and all the identification results corroborated with the phenotypic method for identification. Cassagne C et al reported $87 \%$ correct identification of the clinical isolates by MALDI-TOF MS. ${ }^{13}$ Although there were nonidentification of filamentous fungi in our study but there was no misidentification. Lau AF et al also did not report any misidentification in their study. ${ }^{7}$ However; Cassagne et al reported one misidentification of Rhizopus oryzae as Mucor circinelloides. ${ }^{13}$

Out of 36 Aspergillus isolates, 29 (80.5\%) could be identified. All the identified Aspergillus species showed $100 \%$ corroboration with identification results by conventional method. There were few Aspergillus species whose speciation could be done by Slide culture only. In such cases the species identification could be done early using MALDI-TOF Vitek MS resulting in timely management of patients.

Amongst Zygomycetes, $50 \%$ of the isolates could be identified by MALDI TOF Vitek MS. Rhizomucor pussilus could not be identified by phenotypic method but by MALDI TOF Vitek MS after analysis of the spectra by the SARAMIS database for Research use. This isolate was confirmed as Rhizomucor pussilus by reference laboratory also. We believe that the non-identification of Syncephalastrum racemosum would be because of lack of reference spectra in the database.

Phaeohyphomycetes like Exserohilum rostratum and Scedosporium apiospermum could not be identified although their reference spectra are present in Vitek MS database. However, Cladophialophora bantiana was rapidly identified by MALDI-TOF Vitek MS. The phenotypic identification of Phaeoid fungi is difficult, time consuming and usually requires slide culture for the definitive identification. The isolate of Cladophialophora bantiana was isolated from brain abscess and the rapid identification of the isolate resulted in timely and accurate management of the patient. Lau AF et al could not identify Cladophialophora sp. by MALDI-TOF because of the fact that the reference spectra created for this fungus contained lesser number of protein peaks in comparison to other group of fungi. ${ }^{7}$
There was one isolate of Dermatophyte which was identified as Microsporum gypseum/cookie by phenotypic method but this could not be detected by MALDI-TOF Vitek MS. This can be because of the lack of Microsporum cookie spectra in the database. The accuracy of MALDITOF MS for the identification of Dermatophytes have been reported between 13.5 and $100 \% .^{17}$

Most of the clinical isolates in our study were identified by using extraction protocol-1. The three isolates namely Aspergillus nidulans, Rhizopus arrhizus, Alternaria alternata could be identified only after use of extraction protocol-2. This protocol was the modified version of extraction protocol used for the identification of Phaeoid fungi by Singh A et al..$^{15}$

The utility of MALDI TOF MS has been found to be enormous for the difficult to identify filamentous fungi like Dermatophytes, Phaeohyphomycetes, and various species of Zygomycetes etc. MALDI TOF MS has been found to be not only rapid but economical and significantly less labor intensive for the identification of such type of filamentous fungi.

The two important factors which contribute to the identification by MALDI-TOF MS are appropriate extraction method and robust and reliable library. In our study, the reason for non-identification of filamentous fungi could be inadequate extraction of proteins from filamentous fungi and/or analysis of the test spectra generated by MALDI-TOF with our isolates by utilization of reference spectra library created by the manufacturer. The authors of the studies done for the identification of the filamentous fungi by MALDI-TOF have first created their own library in MALDI-TOF MS using their own isolates and have utilized their in-house library for the analysis of the test spectra. ${ }^{2,13,16,18}$

\section{Conclusion}

Identification of filamentous fungi by MALDI-TOF Vitek MS is a great challenge for a diagnostic laboratory. At the same time, it is important to identify these fungi accurately and timely up to species level for the optimum management of the patients. Conventional methods are time consuming and many-a times the correct identification cannot be done. Molecular methods are a great hope for their identification but these assays have their own limitations like they are expensive and there is a great chance of contamination because of fungal spores. Also, Molecular assays are not developed for the identification of all types of filamentous fungi. DNA sequencing is the only molecular method by which identification of all type of filamentous fungi can be done but it is very expensive, is good for research 
laboratory but is practically difficult to use in a clinical laboratory. We could identify good number of filamentous fungi including Phaeoid fungi and Zygomycetes (70\%) at our institute by utilizing MALDI TOF Vitek MS using database incorporated by the manufacturer. So, based on our study, we anticipate that by standardizing the extraction protocol for filamentous fungi and creating our own inhouse library, MALDI-TOF Vitek MS which is now being used routinely for the identification of bacteria and yeasts can be economically utilized for the rapid and reliable identification of filamentous fungi in a clinical mycology laboratory.

\section{Acknowledgements}

Professor (Dr) Arunaloke Chakraborty, PGIMER, Chandigarh

\section{References}

1. Procop GW, Koneman EW, Curch DL, et al. Koneman's Color Atlas and Textbook of Diagnostic Microbiology.7th international edn. United States of America: Lippincott Williams and Wilkins, 2016.

2. Becker PT, de Bel A, Martiny D, et al. Identification of filamentous fungi isolates by MALDI-TOF mass spectrometry: clinical evaluation of an extended reference spectra library. Med Mycol 2014;8:826-34.

3. Iriart X, Laverne RA, Fillaux J, et al. Routine identification of medical fungi by the new Vitek MS Matrix assisted laser desorption ionisation -time of flight system with a new time effective strategy. J Clin Microbiol 2012;50:2107-10.

4. Chakrabarti A, Singh R. The emerging epidemiology of mould infections in developing countries. Curr Opin Infect Dis 2011;24:521-6.

5. Marr KA, Carter RA, Crippa F, Wald A, Corey L. Epidemiology and outcome of mould infections in hematopoietic stem cell transplants recipients. Clin Infect Dis 2002;34:909-17.

6. Gautier M, Ranque S, Normand AC, et al. Matrix Assisted Laser Desorption Ionisation- Time of Flight Mass Spectrometry: revolutionizing clinical laboratrory diagnosis of mould infection. Clin Microbiol Infect 2014;20:1366-71.

7. Lau FA, Drake SK, Calhoun LB, Henderson CM and Zelanzy AM. Development of a clinically comprehensive database and a simple procedure for identification of moulds from solid media by Matrix Assisted Laser Desorption Ionisation -Time of Flight Mass Spectrometry. J Clin Microbiol 2013;51:828-34.
8. Vidal CG, Upton A, Kirby KA, Marr KA. Epidemiology of invasive mould infections in allogenic stem cell transplant recipients: biological risk factors associated for infection according to time after transplantation. Clin Infect Dis 2008;47:1041-50.

9. Pagano L, Akova M, Dimopoulos G, Herbrecht R, Drgona L, Blijlevens N. Risk assessment and prognostic factors for mould related diseases in immunocompromised patients. J Antimicrob Chemother 2011;66:i5-i14.

10. Normand AC, Cassagne C, Ranque $S$,et al. Assessment of various parameters to improve MALDI -TOF MS reference spectra libraries constructed for the routine identification of filamntous fungi. BMC Microbiol 2013;13:pg76.

11. Schulthess B, Lendermann R, Mouttet F, et al. Use of the Bruker MALDI Biotyper for the identification of molds in the clinical mycology laboratory. J Clin Microbiol 2014;52:2797-803

12. Levesque S, Dufresne PJ, Soualhine H, et al. A side by side comparison of Bruker Biotyper and Vitek MS:Utility of MALDI-TOF MS technology for microorganisms identification in a public health reference laboratory. PLOS ONE $2015 ; 10(12): \mathrm{e} 0144878$.

13. Cassagne C, RanqueS, Normand AC, et al. Mould routine identification in the clinical laboratory by Matrix Assisted Laser Desorption Ionisation -Time Of Flight Mass Spectrometry. PLOS ONE 2011;6:2.

14. Rahi P, Prakash O, Shouche YS. Matrix Assisted Laser Desorption /Ionization Time of Flight Mass Spectrometry (MALDI-TOF MS) Based microbial identifications: Challenges and scopes for microbial ecologists. Front Microbiol 2016; 7:1359.

15. Singh A, Singh PK, Kumar A, et al. Molecular and matrixassisted laser desorption ionization-time of flight mass spectrometry based characterization of clinically significant melanized fungi in India. J Clin Microbiol 2017;55:1090 -103 .

16. De Carolis E, Posteraro B ,Vella A, etal. Species identification of Aspergillus, Fusarium and Mucorales with direct surface analysis by matrix assisted laser desorption ionization- time of flight mass spectrometry. Clin Microbiol Infect 2012;18:475-84.

17. L'Ollivier C, Ranque S. MALDI-TOF-Based Dermatophyte Identification. Mycopathologia 2017;182:183-92.

18. Ranque S, Normand AC, Cassagne C, et al. MALDI-TOF MS mass spectrometry identification of filamentous fungi in the clinical laboratory. Mycoses 2013;57:135-40.

*Corresponding author:

Dr Hena Butta, Department of Microbiology, Indraprastha Apollo Hospitals, New Delhi INDIA

Phone: +91 8447233605

Email: henavasdeva@yahoo.com

Financial or other Competing Interests: None. 